\title{
Atomic scale lattice distortions and domain wall profiles
}

\author{
K. H. Ahn, T. Lookman, A. Saxena, and A. R. Bishop \\ Theoretical Division, Los Alamos National Laboratory, Los Alamos, New Mexico 87545
}

\begin{abstract}
We present an atomic scale theory of lattice distortions using strain related variables and their constraint equations. Our approach connects constrained atomic length scale variations to continuum elasticity and describes elasticity at several length scales. We apply the approach to a twodimensional square lattice with a monatomic basis, and find the elastic deformations and hierarchical atomic relaxations in the vicinity of a domain wall between two different homogeneous strain states. We clarify the microscopic origin of gradient terms, some of which are included phenomenologically in Ginzburg-Landau theory, by showing that they are anisotropic.

PACS numbers: 81.30.-t, 61.50.Ah, 62.20.Dc, 63.20.-e
\end{abstract}

An understanding of atomic scale lattice distortions is essential for correctly describing the elastic energies of nano-structured materials. New generations of experimental tools to probe individual atoms and local environments [1], and the growing interest in complex functional materials, in which local lattice distortions are coupled to electronic, magnetic, and chemical degrees of freedom, further emphasize the need for a consistent theoretical framework to describe strain-based materials. For example, in perovskite manganites the change in oxygen ion displacement at each site is associated with the charge and orbital ordering states [2]. An atomic scale description of the interface or domain wall between two different homogeneous states is thus a first step towards predicting functionality located at the domain walls.

Strain variables (rather than displacement) with constraints have been recently shown to have advantages for describing the long wavelength lattice distortions observed in, for example, martensitic materials and, more generally, solid-solid phase transformations [3]. The anisotropic long-range interaction in the order parameter strain fields drives the formation of a rich landscape of multiscale elastic textures. The aim of this work is to formulate a microscopic description of elasticity and demonstrate the relationship with and differences from long-wavelength continuum theory. We introduce appropriate inter-cell and intra-cell distortion modes and show how the form of the elastic energy recovers the correct phonon spectra. The discreteness of the lattice, choice of modes and constraints among them give rise to an anisotropic gradient expansion for the elastic energy. This leads to elastic domain wall solutions that are different from those predicted using continuum theory; we obtain $0^{\circ}$ and $90^{\circ}$ 'staircase' domain walls for sufficiently small bulk modulus (or 'soft') materials, in addition to the $45^{\circ}$ or $135^{\circ}$ walls predicted from continuum theory for 'hard' materials.

Models based on displacement variables with pairpotentials, such as Born-von Kármán models [4], have been widely used to incorporate 'microscopic elasticity'. However, essentially because distortion implies strain, the physical insight for atomic scale elasticity will reveal itself in the language of strain-related variables presented here. Moreover, our work is quite distinct from recent efforts to describe elasticity of nanometer-sized objects [5]. The interest there is to describe long wavelength strains in a given dimension with other dimensions maintained at nanoscales, such as ultra-thin long nanowires. Our emphasis is to describe atomic scale distortions, irrespective of whether the region of interest is in bulk or nano-sized objects. Our approach describes elastic deformation in terms of intra-cell modes or "shuffles" of atoms, which are essential in describing short wavelength lattice distortions, and distortion of unit cells, instead of adopting coarse graining approximations [6].

We illustrate our ideas in detail for the simplest case, namely a square lattice in two-dimensional (2D) space with a monatomic basis. We find that the most convenient strain-related variables for atomic scale distortions are the normal distortion modes (more precisely, symmetry coordinates) of an elementary square object of four atoms, as shown in Fig. 1] Because of the number of atoms in this object and the dimensionality, eight normal modes exist. The rigid rotation and the two rigid translations (not shown in Fig. 1) cost no elastic energy, and therefore, are not distortion modes. The first three distortion modes in Fig. 1 correspond to the usual dilatation $\left(e_{1}\right)$, shear $\left(e_{2}\right)$, and deviatoric $\left(e_{3}\right)$ strains of the continuum elasticity theory for a square lattice [3]. The next two degenerate modes in Fig. 1] $s_{+}$and $s_{-}$, correspond to the "intracell" or "shuffle" modes of the square lattice [], which are absent in continuum elasticity theory. Our approach uses these five distortion variables defined for each plaquette of four atoms at $\vec{i}, \vec{i}+(10), \vec{i}+(11)$, and $\vec{i}+(01)$, where $\vec{i}$ represents the coordinate of the lattice points, to describe the elastic energy [8].

Since the five variables are derived from two displacement variables for each lattice site, they are related by three constraint equations. By representing $e_{1}, e_{2}, e_{3}$, $s_{+}$, and $s_{-}$in terms of displacement variables $d^{x}$ and $d^{y}$ in $k$ (wavevector) space and eliminating $d^{x}$ and $d^{y}$, the constraint equations are obtained. One of them is the microscopic elastic compatibility equation, which relates strain modes: 


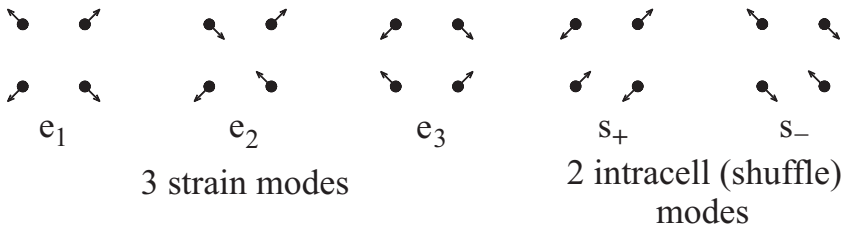

FIG. 1: Normal distortion modes for a square object of four atoms in $2 \mathrm{D}$.

$$
\begin{aligned}
& \left(1-\cos k_{x} \cos k_{y}\right) e_{1}(\vec{k})-\sin k_{x} \sin k_{y} e_{2}(\vec{k}) \\
& +\left(\cos k_{x}-\cos k_{y}\right) e_{3}(\vec{k})=0 .
\end{aligned}
$$

The other two relate the intracell and the strain modes:

$$
\begin{aligned}
& 2 \cos \frac{k_{x}}{2} \cos \frac{k_{y}}{2} s_{ \pm}(\vec{k}) \mp i \sin \left(\frac{k_{x} \pm k_{y}}{2}\right) e_{1}(\vec{k}) \\
& \pm i \sin \left(\frac{k_{x} \mp k_{y}}{2}\right) e_{3}(\vec{k})=0 .
\end{aligned}
$$

These constraints generate anisotropic interactions (reflecting the lattice symmetry) between atomic scale strain fields, similar to the compatibility equations in Ref. [3], but now including the intracell modes. In the long wavelength limit, our description approaches the continuum model: For $\vec{k} \rightarrow 0$, the above constraint equations can be written in real space as

$$
\begin{aligned}
& \nabla^{2} e_{1}(\vec{r})-2 \nabla_{x} \nabla_{y} e_{2}(\vec{r})+\left(\nabla_{y}^{2}-\nabla_{x}^{2}\right) e_{3}(\vec{r})=0 \\
& s_{ \pm}(\vec{r})=\left[\left(\nabla_{y} \pm \nabla_{x}\right) e_{1}(\vec{r})+\left(\nabla_{y} \mp \nabla_{x}\right) e_{3}(\vec{r})\right] / 4 .
\end{aligned}
$$

Equation (3) is the usual compatibility equation in continuum theory. Equation (4) shows that the spatial variations of strains always generate intracell modes, the magnitude of which vanish as the inverse of the length scale of the strain mode variations. It is well-known in continuum Ginzburg-Landau theory that the energy associated with the gradient of strains is responsible for domain wall energies as, for example, in structural phase transitions [7]. The above result shows that the intracell modes are at the origin of such energy terms. Since our strain-related variables become identical to conventional strain variables in the long wavelength limit, various length scale lattice distortions may be described in a single theoretical framework. This makes it possible to study typical multiscale situations where both shortand long-wavelength distortions are important. It also provides a natural framework for incorporating interactions between atomic scale strain-related fields coupled to other degrees of freedom in functional materials.

The following analysis of the simple harmonic elastic energy for the square lattice further exemplifies the utility of these variables. We consider the simplest energy expression by approximating the total elastic energy by the sum of the elastic energy of each square:

$$
E_{\text {sq.lat }}=\sum_{\vec{i}}\left\{\sum_{n=1,2,3} \frac{1}{2} A_{n}\left[e_{n}(\vec{i})\right]^{2}+\sum_{m=+,-} \frac{1}{2} B\left[s_{m}(\vec{i})\right]^{2}\right\}
$$

where $A_{n}$ and $B$ denote elastic moduli and 'intracell modulus', respectively. The couplings between $e_{1}, e_{2}, e_{3}, s_{+}$, and $s_{-}$at the same site are forbidden by symmetry at the harmonic level, but are allowed at the anharmonic (a)

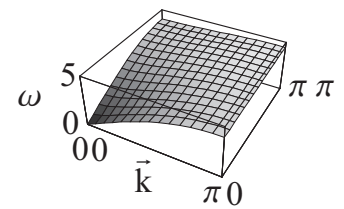

With intracell modes (b)

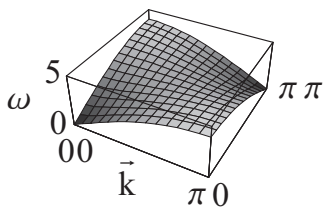

Without intracell modes
FIG. 2: An example of calculated phonon spectra (a) with and (b) without intracell modes for a $2 \mathrm{D}$ square lattice with a mono-atomic basis. The upper phonon branch is shown for both cases $(M=\hbar=1)$.

level, which may have important consequences for structural "phase transitions" at the nanoscale. In Eq. (5) the inter-atomic elastic energies between atoms beyond each square, or further than the second nearest neighbors, are neglected. These interactions may be included by adding energy terms with distortion variables at different sites, e.g., $e_{1}(\vec{i}) e_{1}(\vec{i}+(10))$. Since some of the atomic pairs are shared by two square plaquettes of atoms, the parameters in Eq. (5) should be appropriately renormalized. A robust way to determine the parameters is to compare the phonon spectrum of our model with experimental data.

For the lattice energy of Eq. (5), the phonon spectrum is given by $\sqrt{M} \hbar \omega=\sqrt{E_{1} \pm \sqrt{E_{2}}}$, where $E_{1}=\left(A_{1}+\right.$ $\left.A_{2}+A_{3}\right)\left(1-\cos k_{x} \cos k_{y}\right) / 2+B\left(1-\cos k_{x}\right)\left(1-\cos k_{y}\right)$, $E_{2}=\left(A_{1}+A_{2}-A_{3}\right)^{2} \sin ^{2} k_{x} \sin ^{2} k_{y} / 4+\left(A_{1}-A_{2}+\right.$ $\left.A_{3}\right)^{2}\left(\cos k_{x}-\cos k_{y}\right)^{2} / 4$, and $M$ is the mass of an atom. A typical spectrum (upper branch) for $A_{1}=5, A_{2}=4$, $A_{3}=3$, and $B=5$ is shown in Fig. 22(a). At $\vec{k}=(\pi, \pi)$, the distortion is a pure intracell mode, and the energy depends only on the intracell mode modulus $B$. Therefore, as shown in Fig. 22 (b), $\omega(\pi, \pi)$ vanishes without the intracell mode $(B=0)$, which is unphysical. As $k \rightarrow 0$, the slope of the phonon spectrum is determined only by strain mode moduli, $A_{1}, A_{2}$, and $A_{3}$, whereas the curvature depends on $B$ as well because of Eq. (4). For $k \rightarrow 0$, since the intracell modes vanish as the inverse of the wavelength, the lattice energy approaches $E_{\text {sq.lat }}(\vec{k} \rightarrow 0) \approx \sum_{\vec{k}, n=1,2,3} A_{n} e_{n}^{2}(\vec{k}) / 2$, in agreement with continuum theory. Using Eq. (4), the energy for the intracell modes in Eq. (5) can be written as $E_{\text {intra }} \approx$ $\int d \vec{r} B\left[\left(\vec{\nabla} e_{1}\right)^{2}+\left(\vec{\nabla} e_{3}\right)^{2}+2\left(\nabla_{y} e_{1} \nabla_{y} e_{3}-\nabla_{x} e_{1} \nabla_{x} e_{3}\right)\right] / 16$.

We compare our approach to a displacement-based Born-von Kármán model [4] for the square lattice. The first-nearest and the second-nearest neighbor atoms are connected by central-force and non-central-force springs with spring constants $K_{1}^{C}, K_{1}^{N}, K_{2}^{C}$ and $K_{2}^{N}$, respectively. Elastic energies of the lattice, one from our model and the other from the Born-von Kármán model, can be represented in the following form in terms of displacement variables, $d^{x}(\vec{i})$ and $d^{y}(\vec{i}): \quad E_{\text {sq.lat }}=$ $\sum_{\vec{k}, a, b} d^{a}(-\vec{k}) D_{a b}(\vec{k}) d^{b}(\vec{k})$. We find that the $D_{a b}(\vec{k})^{\prime} \mathrm{s}$ for both models become identical if: $A_{1}=K_{1}^{C}-K_{1}^{N}+$ $2\left(K_{2}^{C}-K_{2}^{N}\right), A_{2}=2\left(K_{1}^{N}+K_{2}^{C}+K_{2}^{N}\right), A_{3}=K_{1}^{C}+$ 
$K_{1}^{N}+4 K_{2}^{N}$, and $B=K_{1}^{C}+K_{1}^{N}$.

We apply our formalism to obtain the domain wall solution for the atomic displacements between two homogeneous strain states (a "twin boundary") due to a phase transition to a rectangular lattice. We then compare the solution to that obtained from continuum theory where discreteness effects are neglected [7]. With elastic energy $E_{\text {rec }}=E_{\text {rec }}^{(1)}+E_{\text {rec }}^{(2)}$, where

$$
\begin{aligned}
& E_{\text {rec }}^{(1)}=\sum_{\vec{i}} \frac{1}{2} A_{1} e_{1}(\vec{i})^{2}+\frac{1}{2} A_{2} e_{2}(\vec{i})^{2}+\frac{1}{2} B\left[s_{+}(\vec{i})^{2}+s_{-}(\vec{i})^{2}\right], \\
& E_{\text {rec }}^{(2)}=\sum_{\vec{i}}-\frac{1}{2} A_{3}^{\prime} e_{3}(\vec{i})^{2}+\frac{1}{4} F_{3} e_{3}(\vec{i})^{4},
\end{aligned}
$$

the degenerate ground state of $E_{\text {rec }}$ is a uniform state with $e_{3}= \pm \sqrt{A_{3}^{\prime} / F_{3}}$, and $e_{1}=e_{2}=s_{+}=s_{-}=0$. To study the domain wall between these two degenerate rectangular ground states, we consider $e_{3}(\vec{i})$ as the order parameter and minimize $E_{\text {rec }}^{(1)}$ with respect to the other variables using the constraint equations [Eqs. (1) and (2)] and the method of Lagrange multipliers. We obtain $E_{\text {rec,min }}^{(1)}=\sum_{\vec{k}} \frac{1}{2} e_{3}(-\vec{k}) U(\vec{k}) e_{3}(\vec{k})$, where $U(\vec{k})=\left(V_{1}+\right.$ $\left.V_{2}+V_{3}\right) / V_{4}$, and $V_{1}=\left[A_{2}\left(A_{1} \beta_{2}^{2}+A_{2} \beta_{1}^{2}\right)+B^{2} \beta_{4}^{2}\right] A_{1} \beta_{3}^{2}$, $V_{2}=\left(2 A_{1} A_{2} \beta_{1}^{2}+A_{2}^{2} \beta_{1}^{2}+A_{1}^{2} \beta_{2}^{2}\right) B \beta_{1} \beta_{4}, V_{3}=2\left(A_{1} \beta_{2}^{2}+\right.$ $\left.A_{2} \beta_{1}^{2}\right) B^{2} \beta_{4}^{2}+B^{3} \beta_{1} \beta_{4}^{3}, V_{4}=\left(A_{1} \beta_{2}^{2}+A_{2} \beta_{1}^{2}+B \beta_{1} \beta_{4}\right)^{2}$, with $\beta_{1}=1-\cos k_{x} \cos k_{y}, \beta_{2}=-\sin k_{x} \sin k_{y}, \beta_{3}=$ $\cos k_{x}-\cos k_{y}$, and $\beta_{4}=\left(1-\cos k_{x}\right)\left(1-\cos k_{y}\right)$.

With $k_{x}=k \cos \theta$ and $k_{y}=k \sin \theta$, the expansion of $U(k, \theta)$ about $k=0$ yields $U(k, \theta)=U_{0}(\theta)+U_{2}(\theta) k^{2}+$ $O\left(k^{4}\right)$, where $U_{0}(\theta)=A_{1} A_{2} \cos ^{2} 2 \theta /\left(A_{1} \sin ^{2} 2 \theta+A_{2}\right)$, and $U_{2}(\theta)=\sin ^{2} 2 \theta\left[6 A_{1} A_{2} B \sin ^{2} 2 \theta+4 A_{1} A_{2}\left(A_{1}+\right.\right.$ $\left.\left.A_{2}\right) \cos ^{2} 2 \theta+3 B\left(A_{2}^{2}+A_{1}^{2} \sin ^{2} 2 \theta\right)\right] /\left[24\left(A_{2}+A_{1} \sin ^{2} 2 \theta\right)^{2}\right]$. The term $U_{o}$ is purely orientation-dependent without a length scale, and is minimized at $\theta=45^{\circ}$ and $135^{\circ}$, as obtained in Ref. [3]. The difference between continuum and our discrete theory lies in the $k^{2}$ term: continuum theory commonly assumes isotropic gradients in the order parameter, i.e., $\left(\vec{\nabla} e_{3}\right)^{2}$ [] , whereas $U_{2}(\theta)$ is anisotropic. The two origins of the anisotropy are: (a) the compatibility relation, Eq. (1), which has higher powers in $k$ than Eq. (3) due to discreteness, and (b) the presence of shuffle mode energy. The latter can be written as gradients of strains, but with corrections to the phenomenological isotropic term, $\left(\vec{\nabla} e_{3}\right)^{2}$, used in Ginzburg-Landau theory. As $U_{2}(\theta)$ is minimized for $\theta=0^{\circ}$ and $90^{\circ}$, it competes with $U_{0}(\theta)$ which prefers $\theta=45^{\circ}$ and $135^{\circ}$. Thus, the domain wall direction depends on the length scale with a critical length scale $\lambda_{c} \sim \sqrt{B / A_{1}}$. If $\lambda_{c} \leq 1$, i.e., less than the interatomic spacing, the domain wall has direction $45^{\circ}$ or $135^{\circ}$ down to atomic scales. If $\lambda_{c}>1$, then for length scales smaller (larger) than $\lambda_{c}$, the domain wall direction is $0^{\circ}$ or $90^{\circ}\left(45^{\circ}\right.$ or $\left.135^{\circ}\right)$ and the domain wall has multiscale attributes.

We examine first the case $\lambda_{c} \leq 1$ that would apply to materials with relatively large bulk modulus $A_{1}$ ('hard' materials) for fixed $B$. Here $k_{x}= \pm k_{y}$ and $U(\vec{k})=B\left(1-\cos k_{x}\right) /\left(1+\cos k_{x}\right)$. The domain wall width is a result of the competition between $U(k)$ that

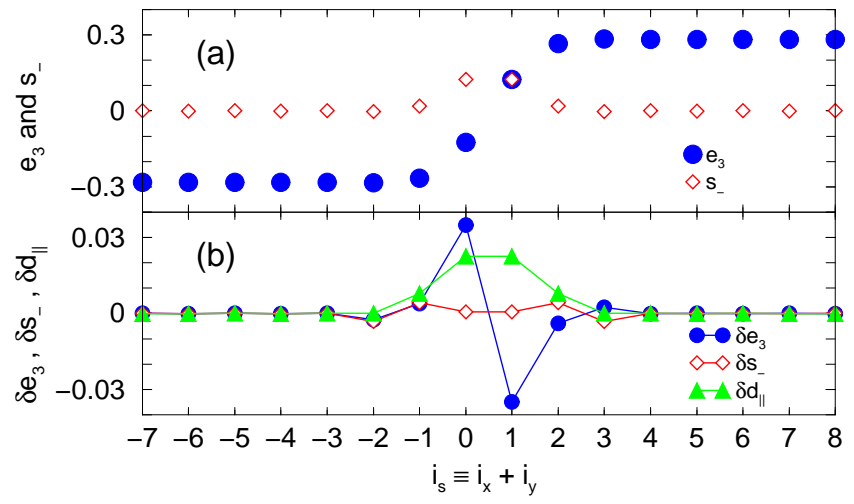

FIG. 3: (Color) Atomic scale $135^{\circ}$ domain wall profile for critical length scale, $\lambda_{c} \leq 1$ along the direction perpendicular to the domain wall: (a) strain $e_{3}$ and shuffle $s_{-}$, (b) differences in $e_{3}\left(\delta e_{3}=e_{3, \text { atomic }}-e_{3, \text { continuum }}\right), s_{-}\left(\delta s_{-}\right)$and displacement parallel to the domain wall direction $\left(\delta d_{\|}\right)$between the results from continuum theory for $\vec{k} \sim 0$ and our model that includes discreteness. The fields $e_{1}, e_{2}, s_{+}$, and displacement perpendicular to the domain wall are zero. Parameter values are $A_{1}=5, A_{2}=4, A_{3}^{\prime}=4, B=5$, and $F_{3}=50$.

favors $\vec{k} \rightarrow 0$, or thick domains, and $E_{\text {rec }}^{(2)}$ that favors sharp walls. We illustrate the domain wall solution with $135^{\circ}$ domain wall direction. The only non-zero distortion modes are $e_{3}$ and $s_{-}\left(s_{+}\right.$for a $45^{\circ}$ domain wall). The strain $e_{3}$ reverses sign at the domain wall, the intracell mode $s_{-}$is confined within the domain wall, and the atomic displacements are parallel to the domain wall direction. The numerical solution [9] for $e_{3}$ and $s_{-}$along a line perpendicular to the wall is shown in Fig. B(a), for which $\lambda_{c} \sim 1$. (Narrow domain walls with widths of a few unit cells, as considered here, have been identified experimentally [10].) The corresponding displacement field near the center of the domain wall is shown in Fig. 4(a), in which the red and blue colors show regions with $e_{3}$ positive and negative, respectively. Both figures show that the center of the domain wall is located at bonds rather than sites to avoid the higher energy state of $e_{3}=0$ and large $s_{-}$. As for a Peierls-Nabarro barrier [11], the higher energy (by $4.4 \times 10^{-4}$ per unit length for our parameter values) for the site-centered domain wall acts as a pinning potential for the domain wall due to the inherent discreteness. In Fig. 3(b) we compare our results with continuum theory, which predicts $e_{3}=e_{3}^{\max } \tanh \left(i_{s} / \xi\right)$ [] $]$ and $s_{-}=\partial e_{3} / 2 \partial i_{s}$ from Eq. (4), where $i_{s}=i_{x}+i_{y}$. The differences in the interface region, shown in Fig. B(b), are of the order of $10 \%$ of $e_{3}^{\max }=\sqrt{A_{3}^{\prime} / F_{3}}$. The domain wall width [10] is roughly given by $2 \xi=\sqrt{2} \sqrt{B / A_{3}^{\prime}}$ and the ratio between the maxima of $s_{-}$and $e_{3}, s_{-}^{\max } / e_{3}^{\max }$, is about $1 /(2 \xi)$.

Anisotropic effects in $U(k, \theta)$ become more apparent away from equilibrium, e.g., at finite temperatures or in other conditions where metastability is present. Fig- 


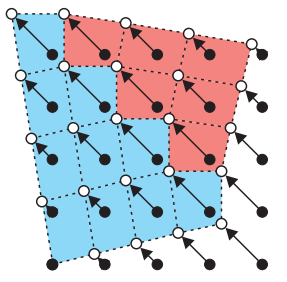

(a)

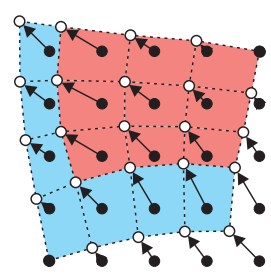

(b)

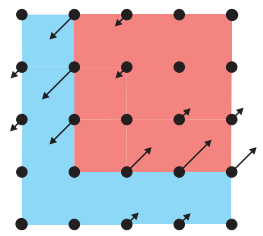

(c)
FIG. 4: (Color) Atomic displacements in the vicinity of domain wall: (a) $\lambda_{c} \leq 1$ and (b) $\lambda_{c}>1$. Color represents the sign of $e_{3}$ (red: positive, blue: negative), and is lighter compared to Figs. [5 and 6] to make the arrows visible. (c) The displacement component perpendicular to the large scale domain wall direction for (b), magnified by a factor of 3 . Parameter values are $A_{2}=4, A_{3}^{\prime}=4, B=5, F_{3}=50$, and $A_{1}$ $=5$ for (a) and $A_{1}=1$ for (b) and (c).
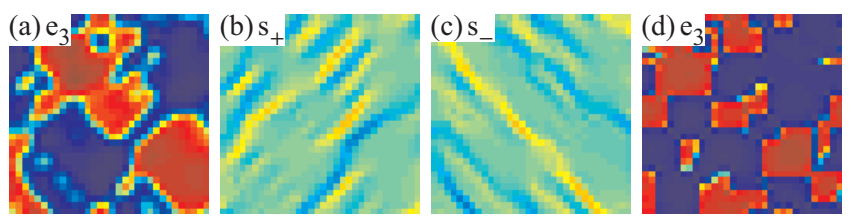

FIG. 5: (Color) Nonequilibrium domain wall state: (a) $e_{3}$, (b) $s_{+}$, (c) $s_{-}$for $\lambda_{c} \leq 1$, (d) $e_{3}$ for $\lambda_{c}>1$. Parameter values are identical to Fig. 4 Dark red corresponds to 0.28 and dark blue to -0.28 . Green implies a value close to zero.

ure 5(a) shows the results for $e_{3}$ of a $2 D$ simulation away from equilibrium 12, in which atomic scale domain walls are oriented along $45^{\circ}$ and $135^{\circ}$ directions. The corresponding intracell modes are shown in Figs. 5(b) and 5(c). Note that both $s_{+}$and $s_{-}$shuffles are present at or near interfaces and only one of these modes survives at equilibrium. The horizontal or vertical "jogs" in the $s_{+}$and $s_{-}$walls are secondary defects due to the competition between $U_{0}$ and $U_{2}$, which provide principal relaxation forces.

The domain wall solution for $\lambda_{c}>1$, typical for small bulk modulus $A_{1}$ or 'soft' materials, is shown in Fig. 6 for which $\lambda_{c} \sim \sqrt{5}$. The $e_{3}$ field in Fig. 6(a) shows that on length scales of the size of the system (larger than $\left.\lambda_{c}\right)$, the diagonal orientation is still preferred. However, this diagonal domain wall consists of a 'staircase' of $0^{\circ}$ and $90^{\circ}$ domain walls of length scale $\lambda_{c}$. The existence of $0^{\circ}$ and $90^{\circ}$ walls in $e_{3}$ forces elastic compatibility to induce alternately large positive and negative values in the dilatation strain $e_{1}$ in the horizontal and vertical parts of the 'staircase', as shown in Fig. [6(b). This has implications for the functionality of the domain walls. For example, the $e_{1}$ field can couple to charge and modulate the local charge density along the wall. Similar features are also reflected in $s_{+}$and $s_{-}$, as shown in Figs. [6 (c) and 6 (d). The displacement pattern within the square in Fig. [6 is shown in Fig. [(b). Unlike the case $\lambda_{c} \leq 1$ in Fig. $4(\mathrm{a})$, the displacement has a component perpendic-
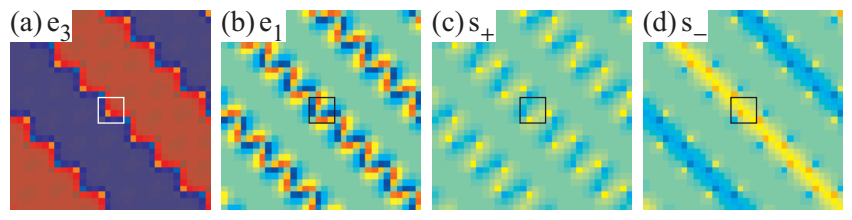

FIG. 6: (Color) Atomic scale domain wall solution for materials with $\lambda_{c}>1$. Parameters are the same as in Figs. 4(b) and 4(c), which show the region inside the square in this figure. Strain $e_{2}$ is zero. Color scheme is the same as in Fig. [5]

ular to the $135^{\circ}$ large scale domain wall direction, which is shown in Fig. 目(c) magnified by a factor of 3. This component is greatest for the atoms at the boundary of the two domains. A nonequilibrium state is shown in Fig. 5(d). The small domains have $0^{\circ}$ or $90^{\circ}$ boundaries, but over larger length scales these domains are correlated along $45^{\circ}$ and $135^{\circ}$ directions.

In summary, we have reported an approach to "atomicscale elasticity", which uses symmetry modes of elementary objects of atoms as distortion variables. A gradient expansion for the energy with anisotropic coefficients has been obtained, with corrections to the usual phenomenological isotropic gradient terms used in Ginzburg-Landau theory. As an illustration, we have obtained domain wall (twin boundary) solutions in terms of strain and intracell modes and have shown how they differ from the continuum elastic soliton solution [7]. Our work provides the basis for interpreting atomic scale features in HREM studies of domain walls [1, 10].

We thank A. J. Millis and S. R. Shenoy for insightful discussions. This work was supported by the US DOE.

[1] S. Stemmer et al., Phil. Mag. A 71, 713 (1995).

[2] A. J. Millis, Phys. Rev. B 53, 8434 (1996); P. G. Radaelli et al., ibid. 55, 3015 (1997).

[3] S. R. Shenoy et al., Phys. Rev. B 60, R12 537 (1999); T. Lookman et al., ibid. 67, 024114 (2003).

[4] C. Kittel, Introduction to Solid State Physics (Wiley, New York, 1960), 2nd ed., p. 96.

[5] J. P. Lu, Phys. Rev. Lett. 79, 1297 (1997); E. Hernández et al., ibid. 80, 4502 (1998); D. E. Segall, S. Ismail-Beigi, and T. A. Arias, Phys. Rev. B 65, 214109 (2002).

[6] I. Goldhirsch and C. Goldenberg, arXiv:cond-mat/0203360

[7] G. R. Barsch and J. A. Krumhansl, Phys. Rev. Lett. 53, 1069 (1984).

[8] E.g., $e_{3}(i)=\left[-d^{x}(i)+d^{y}(i)+d^{x}(i+(10))+d^{y}(i+(10))+\right.$ $\left.d^{x}(i+(11))-d^{y}(i+(11))-d^{x}(i+(01))-d^{y}(i+(01))\right] / 2 \sqrt{2}$. Care needs to be exercised in discretizing derivatives. For example, $e_{3}(i)=\partial d^{x} / \partial x-\partial d^{y} / \partial y \approx\left[d^{x}(i+(10))-\right.$ $\left.d^{x}(i)\right]-\left[d^{y}(i+(01))-d^{y}(i)\right]$ does not reflect the symmetry of the square lattice.

[9] We use the Euler method(see, e.g., Ref. [3]) with an appropriate boundary condition.

[10] J. Chrosch and E. K. H. Salje, J. Appl. Phys. 85, 722 
(1999), and references therein.

[11] F. Nabarro, in Theory of Crystal Dislocations (Clarendon, Oxford, 1967).
[12] These states are found by probing the energy landscape with the method of steepest descent, starting from random initial conditions. 